\title{
CURVA DE PHILLIPS E MACRODINÂMICA DO CAPITAL ÓTIMO*
}

\author{
Fernando Motta Correia ${ }^{* *}$ \\ João Basílio Pereima ${ }^{* * *}$
}

RESUMO: Este artigo apresenta novos argumentos para alguns dos fatos estilizados, recentemente, sobre a dinâmica da inflação, apontados por Mishkin (2007), quais sejam: a) a persistência dos choques de inflação estão declinando; b) a curva de Phillips esta se tornando mais horizontal e; c) a inflação tem se mostrado menos reativa à choques nos preços. É abordado o problema da determinação da oferta agregada em que alegamos que a versão novokeynesiana da curva de Phillips, apesar dos avanços, ainda não incorpora os determinantes da ampliação do produto potencial e, que esta inclusão é uma das explicações para os fatos estilizados (fato b), revelados recentemente por Mishkin.

PALAVRAS-CHAVE: curva de Phillips; inflação; produto potencial

\section{PHILLIPS CURVE AND THE MACRODYNAMICS OF OPTIMAL CAPITAL}

\begin{abstract}
This paper presents new arguments for some recent stylized facts about the dynamics of inflation shown by Mishkin (2007), which are: a) the persistence of inflation shocks are declining; b) the Phillips' curve of this becoming more horizontal and; c) inflation has been less reactive to shocks on prices. It addressed the problem of determining the aggregate supply where the new version of the Keynesian Phillips' curve, that despite progress, not yet incorporating the determinants of the expansion potential of the product and that this inclusion is one of the explanations for stylized facts (fact b ) revealed recently by Mishkin.
\end{abstract}

KEY WORDS: Phillips' curve; inflation; potential output.

JEL: E6; E31; O54.

\footnotetext{
* Artigo recebido em 28/01/2010 e aprovado em 20/06/2011.

** Doutor em Desenvolvimento Econômico e professor adjunto do Departamento de Economia da UFPR. Contato: fmottabr@yahoo.com.br.

${ }^{\star * *}$ Mestre e doutorando em Desenvolvimento Econômico, professor do Departamento de Economia da UFPR. Contato: joaobasiliop@yahoo.com.br.
} 


\section{INTRODUÇÃO}

Em linhas gerais, a concepção teórica consensual na década de 1960 em macroeconomia está associada à interpretação da Teoria Geral de Keynes desenvolvida por Hicks (1937) quando incorporou a relação inversa entre salários nominais (ou inflação) e desemprego descoberta por Phillips (1958) - para o caso inglês, e corroborada por Samuelson e Solow (1960) - para o caso norte-americano. Com a introdução das expectativas na curva de Phillips, reavaliações sobre o comportamento da curva de Phillips, como os trabalhos de Stock e Watson (2007), Cogley e Sargent (2005) e Cecchetti et al. (2007) têm revelado que nos anos recentes, a sensibilidade da inflação ao hiato do produto sofreu uma mudança estrutural, tornando-se menor, ou seja, a curva de Phillips pode estar se tornando mais horizontal.

Mishkin (2007) aponta três fatos estilizados recentemente, sobre a dinâmica da inflação: a) a persistência dos choques de inflação estão declinando; b) a curva de Phillips esta se tornando mais horizontal e; c) a inflação tem se mostrado menos reativa à choques nos preços. Por traz desses fatos, estaria uma mudança importante ocorrida nas economias nos últimos anos que é o fato de que a inflação está ancorada em expectativas racionais do tipo forward looking.

Esses fatos estilizados citados por Mishkin, relacionados com Canadá, Itália, Reino Unido, Alemanha e Japão podem ser explicados dentro da tradição novokeynesiana, a qual assume uma curva de Phillips modificada, que incorpora as expectativas racionais ao mesmo tempo em que mantém os microfundamentos da existência de formas diversas de rigidez. A curva de Phillips novokeynesiana (doravante NKPC) ${ }^{1}$ ajuda a explicar teoricamente os fatos revelados por Mishkin, no entanto alguns avanços no que tange ao ponto $b$ acima, ainda podem ser melhor explicados por novas versões da NKPC.

O objetivo do artigo é propor uma explicação adicional para a horizontalização da curva de Phillips. Em essência, a principal contribuição do artigo está em mostrar que além da ancoragem da inflação por um processo de expectativas racionais do tipo forward looking, a dinâmica da inflação-produto é afetada também pela ampliação da capacidade de produção. Isso contribui para o deslocamento da curva de oferta agregada, mesmo em médio prazo, ao invés de considerar apenas o deslocamento da oferta demanda agregada ao longo da curva, como tradicionalmente ocorre. $\mathrm{O}$ argumento essencial é de que o produto potencial não se expande de forma exógena e constante, mas está condicionado ao estágio do ciclo econômico. Não faz sentido

\footnotetext{
${ }^{1}$ Mantivemos a sigla NKPC, que se refere ao original, new keynesian Phillips' curve.
} 
uma economia expandir sua capacidade de produção na mesma velocidade, em uma recessão, ou caso contrário, em estágio de crescimento acelerado. Em recessões mais profundas a ampliação da oferta pode até ser zero.

Para atingir tal objetivo, o artigo está dividido três seções, além da introdução. A seção dois retoma o histórico da teoria da curva de Phillips dentro da tradição novokeynesiana até chegar à sua versão atual. Na seção três apresentamos um modelo macroeconômico linear nos parâmetros em que introduzimos o efeito capacidade do investimento, o qual tem por resultado conduzir a uma modificação na NKPC, pela introdução de efeito do deslocamento do produto potencial, que é uma das explicações para a horizontalização da curva de Phillips. Por fim na seção quatro reapresentamos as conclusões principais.

\section{A LONGA HISTÓRIA DA CURVA DE PHILLIPS NOVOKEYNESIANA E O PROBLEMA DA OFERTA}

Entre o fim da década de 1960 e o início da década de 1970, com a insuficiência do paradigma da síntese neoclássica em explicar alguns fenômenos econômicos (notadamente a estagflação), uma linha de pensamento econômico se desenvolveu no intuito de entender mais detalhadamente o papel das políticas econômicas no processo de estabilização das flutuações econômicas. A crença tradicionalmente vigente sustentava que as políticas econômicas discricionárias (aquelas que são otimizadas em cada momento do tempo) eram eficazes para afetar o produto real conduzindo-o ao seu nível de pleno emprego, entendido, na linguagem keynesiana, como ausência de desemprego involuntário. Tal conclusão geralmente era obtida a partir da resolução analítica de macromodelos agregados devidamente subsidiados por estudos macroeconométricos que forneciam os parâmetros comportamentais necessários.

Os regimes macroeconômicos observados nos países desenvolvidos, e em desenvolvimento nos últimos 10 anos, apresentam como característica marcante um retorno da validade da curva de Phillips, após o seu abandono no período de estagflação observado em meados dos anos 1970 e durante os anos 1980. Isso implica em uma revigoração da análise macroeconômica baseada nas relações entre taxa de desemprego e taxa de inflação da economia. Esta retomada coincide a forte atuação dos bancos centrais na condução de políticas monetárias estabilizadoras, seja através de sistemas de metas de inflação, seja através de um sistema de intervenção discreta, sem metas declaradas, atuações essas que determinam, em grande medida, o comportamento em curto e médio prazo das variáveis macroeconômicas agregadas reais. Tudo isso, só foi possível graças à recuperação da eficácia da política monetária, o que reavivou por sua vez o antigo debate entre keynesianos e monetaristas, em torno da chamada curva de Phillips novokeynesiana (Taylor, 1979; 1980). 
No entanto, a revigoração recente da curva de Phillips, não se deu nos mesmos moldes das discussões teóricas dos anos 1970, que contrapôs monetaristas e keynesianos, em torno dos efeitos em curto (first round) e em longo prazo (second round) das políticas fiscais e monetárias (Friedman, 1972; Blinder e Solow, 1973; Tobin e Buiter, 1976; Buiter, 1977).

A proposição original da curva de Phillips (1958), baseada em dados estatísticos do Reino Unido $^{2}$, que é o pano de fundo do debate entre monetaristas e keynesianos, demonstrava as relações entre o nível de salário nominal e o nível de emprego. O mecanismo de ajuste ocorre no mercado de trabalho, com os trabalhadores ofertando e as empresas demandando mais ou menos empregos conforme a percepção (expectativas) de cada um em relação às variações no nível de preços. Na versão original da curva de Phillips, existe uma rigidez nominal em curto prazo, e os salários são fixados com base na percepção do desemprego apenas. A ausência de expectativas na versão original decorre pelo fato de que os dois estudos originais (Phillips para UK, Solow e Friedman para os EUA) apontavam para uma inflação média passada próxima ou igual a zero, uma vez que o período coberto pelas séries temporais envolveram os anos 1930, 1940 e 1950, em que se observaram períodos de deflação nessas séries. Como os trabalhadores fixariam salários com base no passado (backward looking) e a média do passado era zero, então suas expectativas apontavam para uma inflação presente também igual a zero, de modo que as expectativas desapareceram. A curva simples de Phillips é então uma relação inversa entre salários e desemprego apenas, que pode ser representada por:

$$
\omega_{t}=-\beta u_{t}+\varepsilon_{t}^{3}
$$

Em meados dos anos 1970, a curva original de Phillips não encontrou mais evidências empíricas, o que levou muitos autores a abandonar sua validade, fato esse que foi antecipado teoricamente por dois trabalhos: Friedman (1968) e Phelps (1968). O comportamento dos preços nos anos 1970 foi marcado por uma persistência das taxas de inflação, de forma que a inflação média passada não era mais zero. A persistência da inflação levou a reformulação da curva de Phillips que passou a incorporar as expectativas passadas. A inflação presente passa a ter um componente expectacional, além da

\footnotetext{
${ }^{2}$ As mesmas evidências foram encontradas para os EUA em seguida por Solow e Samuelson (1960).

${ }^{3} \mathrm{Em}$ que $\mathrm{w}_{t}$ é a taxa de mudança do salário nominal no período $t ; b$ é um parâmetro que mede a sensibilidade dos salários à taxa de desemprego $u$; e $e_{t}$ é um termo aleatório, do tipo ruído branco, i.i.d $\left(0, s^{2}\right)$. Na primeira versão da curva de Phillips não há qualquer tipo de expectativas, e a relação é tratada em termos de salários e desemprego, mais tarde substituída por inflação e diferença entre taxa de desemprego efetiva e taxa de desemprego natural ou friccional.
} 
taxa de desemprego observada. Expectativas do tipo backward looking fixadas de forma rígida ou adaptativa que, no entanto, ao transmitirem o passado para o presente e futuro, podem levar a processos aceleracionistas, pelo simples fato de que choques de oferta aumentam a inflação hoje, e que será usada amanhã para formar as novas expectativas, e assim sucessivamente. Isso levou a reformulação para a chamada curva de Phillips aumentada (pelas expectativas) ou curva de Phillips aceleracionista, cuja forma passou a ser representada como sendo:

$$
\pi_{t}=\alpha E\left(\pi_{t} \mid \pi_{t-1}\right)-\beta\left(u_{t}-u^{*}\right)+\varepsilon_{t} \quad 0 \leq \alpha \leq 1^{4}
$$

A crítica de Friedman e Phelps, no entanto, suscitou reformulações sutis na curva as quais, de certa forma, contribuíram para manter a validade da curva de Phillips através de uma segunda transformação da curva original ${ }^{5}$. A validade da curva foi restabelecida, não para o plano preço salário-desemprego, mas para o plano variação da taxa de inflação-desemprego. A taxa de desemprego não esta relacionada com o nível de inflação (que nos anos 1960 e 1970 foi ascendente), e sim à sua taxa de variação. O que interessa em essência, não é o nível da inflação, mas a aceleração da taxa de inflação, de forma que a política monetária em curto prazo ainda funciona, ao contrário do que havia sido afirmado por Friedman e Phelps.

A curva de Phillips aumentada pelas expectativas (rígidas ou adaptativas) foi um avanço, mas sua versão aceleracionista não condizia com os fatos, gerando assim muito desconforto na teoria econômica. De fato, salvo casos específicos de hiperinflações localizadas, a regra geral era de que o futuro não era uma mera repetição do passado, ou de forma invertida, o passado não é um bom previsor do futuro. Por trás das expectativas passadas está um pressuposto forte de que os agentes não aprendem; pressuposto parcialmente relaxado com a versão adaptacionista das expectativas, em que os agentes corrigem erros cometidos no passado, através da ponderação do erro de previsão calculado como sendo a diferença entre o preço esperado e o verificado em $t-1$. De qualquer forma em ambos os casos os agentes formam expectativas do tipo backward looking.

Uma mudança significativa foi obtida pelos novos clássicos através da introdução das expectativas racionais, como um novo argumento que poderia invalidar a curva de Phillips expandida. Caso isso seja cabalmente demonstrado, diziam os novos clássicos,

\footnotetext{
${ }^{4}$ Em que $\pi_{t}$ é a taxa de inflação observada no período $t ; E\left(\pi_{t} \mid \pi_{t-1}\right)$ é a expectativa de inflação no período $t$ dado a inflação observada no período $t-1 ; \alpha$ é um parâmetro que mede o quanto da inflação passada é assumida na inflação presente; e $u^{*}$ é taxa natural de desemprego. Os demais termos já foram nomeados na nota de rodapé 1 .

${ }^{5}$ A primeira reformulação foi a inclusão de expectativas tipo backward looking, na versão aceleracionista.
} 
então não haveria mais sentido em usar a política monetária e fiscal, mesmo em curto prazo, para justificar intervenções do tipo keynesianas como meio de obter o pleno emprego, pois estaríamos no reino da moeda neutra, novamente.

Embora as expectativas racionais já tenham sido desenvolvidas em 1960-1961 por Muth, foi somente a partir dos trabalhos de Lucas (1972, 1973, 1976) e Sargent e Wallace (1975) que a macroeconomia sofreu toda uma reformulação. Esses trabalhos pioneiros foram a resposta novoclássica para a sobrevivência da curva de Phillips, tão cara aos keynesianos. A principal mudança na teoria macroeconômica adveio da introdução de expectativas do tipo forward looking. Os agentes passam a formar expectativas com base em previsões sobre o comportamento futuro das variáveis. Para fazer essas previsões os agentes usam o conjunto de informações existentes hoje e usam algum modelo racional para prever o comportamento dessa variável no futuro, de forma que, na posse de tais informações, eles não cometerão erros. Se isso for válido, então qualquer mudança nos preços relativos, nas variações da moeda, na taxa de juros ou na política fiscal será antecipada pelos agentes que ajustarão suas expectativas. Os agentes conhecem o ambiente e as relações entre as variáveis, de forma que ajustam continuamente suas expectativas presentes eliminando assim qualquer possibilidade de cometerem erros sistemáticos. O adjetivo racional advém da capacidade ilimitada de processar informações e coordená-las logicamente através de um modelo representativo e fiel à realidade. Graças a esse mecanismo, a única possibilidade da política monetária ou política fiscal influenciar o nível de produto em curto e médio prazo é a possibilidade de causar surpresa aos agentes, o que teria motivado toda uma literatura sobre quando e como isso poderia ocorrer, e se as autoridades monetárias deveriam agir através de discricionariedade de forma que possam surpreender os agentes. A curva de Phillips versão aceleracionista, nesse caso, é novamente modificada pela substituição da expectativa de inflação com base no passado, por um termo expectacional sobre qual será a inflação em $t+1$, a partir de um conjunto de informações sobre os preços $(\pi)$ obtidas no tempo $t$ :

$$
\pi_{t}=\alpha E\left(\pi_{t+1} \mid \pi_{t}\right)-\beta\left(u-u^{*}\right)+\varepsilon_{t} 0 \leq \alpha \leq 1
$$

Com essa reformulação, o mundo clássico da ineficácia da política monetária teria sido restabelecido sob uma roupagem novoclássica baseada nas expectativas racionais. A resposta novokeynesiana não tardou, e veio basicamente através dos argumentos que reafirmam a existência de rigidez nominal de preços. Esses argumentos estão baseados na existência de custos operacionais incorridos pelas empresas para aumentar preços (Mankiw, 1985; Blanchard e Kiyotaki, 1987), em que pequenos "custos de menu" podem conduzir a significativos ciclos econômicos, de forma que microajustes não são possíveis, pois as empresas só consideram viável alterar suas tabelas de preços 
a partir de uma certa magnitude de aumentos de custos. O mark-up, dentro de uma pequena escala pode flutuar devido à um pequeno aumento de custos que não é imediatamente repassado aos preços. Outro argumento novokeynesiano é a existência de contratos que reajustam preços em pontos diferentes do tempo (staggered), de forma que não é possível que haja um ajuste instantâneo de preço do tipo walrasiano (Taylor, 1979, 1980). A reafirmação da curva de Phillips baseada na rigidez de preços, seja por custos, seja por defasagem, seja escalonagem de datas de contratos, feita por Taylor é bem clara:

These rigidities have been remarkably successful in explaining observed correlations between such aggregates as inflation and unemployment, despite the constrains of rational expectations and fixed natural rate of unemployment. Indeed, statistical Phillips curve are an essential property of these models. (Taylor, 1980, p. 1, grifo nosso)

Além dos "custos de menu" e escalonagem de contratos, a rigidez nominal pode ser explicada também por racionalidade limitada (Akerlof e Yellen, 1985) quebrando assim o pressuposto da racionalidade ilimitada presente nos modelos novoclássicos de expectativas racionais. A racionalidade limitada pode ser causada pela incapacidade real da empresa em saber, por exemplo, quais são as decisões que todas as outras empresas estão tomando e em que momento elas estão fazendo isto. Dessa forma é impossível que a empresa em questão possa maximizar qualquer função utilidade ou função de lucro, de forma a obter uma otimização perfeita.

Esse longo debate e percurso trouxeram até o momento atual em que o problema da teoria macroeconômica, dada a validade da curva de Phillips, é determinar as condições em que operam as políticas monetárias e fiscais, isto é, os canais e os efeitos sobre a taxa de desemprego e a taxa de inflação, uma vez que elas não são neutras, como afirmavam os novos clássicos. Esse é o ponto que interessa aqui, e é o ponto que se pretende tratar formalmente, através de um modelo que faz uso da curva de Phillips novokeynesiana, mas com a possibilidade de que o nível de produto potencial possa variar endogenamente, diferentemente do que até então tem sido feito nos modelos novokeynesianos recentes, em que o produto potencial é dado.

Antes de desenvolver os argumentos, julgamos necessário dar mais dois passos no retrospecto da curva de Phillips, para destacar dois pontos importantes: a) como a curva de Phillips com expectativas racionais foi transformada no que se convencionou chamar de curva de Phillips novokeynesiana (NKPC); e b) como a NKCP preserva as características originais de refletir apenas o lado da demanda sem considerações maiores sobre a influência da política econômica sobre o lado da oferta, mesmo em médio prazo. Mesmo usando o conceito de gap, ou hiato do produto, a teoria macroeconômica novokeynesiana restringe-se aos efeitos da demanda agregada prestando pouca atenção aos efeitos da oferta. Este ponto "b" é o nosso ponto de partida. 


\subsection{REGRAS MONETÁRIAS E A CURVA DE PHILLIPS NOVOKEYNESIANA (NKPC)}

A NKPC surge concomitantemente com a reformulação recente sobre os canais da política monetária, e com a mudança da atitude de autoridades monetárias, reconhecendo o fato de que a moeda nas economias capitalistas modernas tem se tornado endógena ${ }^{6}$, em função do surgimento de novos tipos de moedas e aperfeiçoamentos do sistema financeiro, especialmente os mecanismos de concessão de crédito (Stiglitz, 2003). Desta feita a política monetária deve utilizar como instrumento, não o controle do estoque de moeda e indiretamente a taxa de juros, mas o controle direto da própria taxa de juros. Na teoria da política monetária isso significou a substituição da regra de Friedman (1968), que estipulava que a autoridade monetária deveria fixar uma taxa constante de crescimento da moeda como meio de estabilizar a volatilidade do produto e dos preços, pela regra de Taylor (1993), que determina que a taxa de juros deva responder às variações no nível de preços e no produto ao mesmo tempo. A regra original de Taylor, tal como sugerida no artigo de 1993 estabelece que:

$$
r=p+0,5 y+0,5(p-2)+2
$$

Em que $r$ é a taxa nominal de juros a ser estabelecida pela autoridade monetária, $p$ é a taxa de inflação dos últimos quatros trimestres, e $y$ é o desvio em termos percentuais do produto $(Y)$ em relação ao produto potencial $\left(Y^{*}\right)$, isto é, $y=100\left(Y-Y^{*}\right) / Y^{*}$, e o termo 2 é o que Taylor chamou de taxa de juros real de "equilíbrio", a taxa de juros que possibilita que o produto cresça na mesma velocidade do produto potencial. Essa regra pode ser reescrita substituindo seus valores originais por constantes, com o que teríamos:

$$
i_{t}=\pi_{t}+\alpha\left(\pi_{t}-\pi^{*}\right)+\beta y_{t}+r^{*}
$$

Em que $i$ é a taxa de juros nominal; $r^{*}$ é a taxa real de juros de equilíbrio; $\pi$ é a taxa média da inflação observada nos últimos quatro trimestres, assumida como sendo a inflação presente; $\pi^{*}$ é a meta para taxa de inflação; e $y$ é o hiato do produto.

A regra de Taylor para condução da política monetária tem embutida uma curva de Phillips do tipo forward looking, isto é, segue o processo das expectativas racionais:

Why do we need any special treatment of these transitions [transição do nível de emprego após acionada a politica monetária]? First, the research that underlies the design of policy rules assumes that expectations are rational. (Taylor, 1993, p. 207)

${ }^{6}$ A versão horizontalista da moeda de Kaldor (1982) e Moore (1988). 
A equação (iii) mostrou a versão da curva de Phillips estendida, em que o processo expectacional envolve expectativas reacionais. É um processo como este que está por trás do uso de regras monetárias do tipo das regras propostas por Taylor. No entanto a teoria novokeynesiana fez mais uma transformação na curva de Phillips chegando-se então à forma final da NKPC. Esta transformação consiste em substituir a variável desemprego $(u)$ e desemprego natural $\left(u^{\star}\right)$ por seu equivalente produto $(y)$ e produto potencial $\left(y^{*}\right)$. A curva de Phillips novokeynesiana foi originalmente proposta por Calvo (1983), com base em uma estrutura de rigidez de preços e reapresentada por Gali e Gertler (1999), Sbordone (2002) e Mankiw e Reis (2002), em que o leitor interessado pode obter maiores informações sobre sua derivação, especialmente no apêndice do último trabalho. A NKPC, com base em rigidez nominal de preços e expectativas forward looking, tem então, a seguinte forma ${ }^{7}$ :

$$
\pi_{t}=\beta E_{t} \pi_{t+1}+\delta g_{t}+z_{t}
$$

em que $\pi_{t}$ é a taxa de inflação; $E_{t} \pi_{t+1}$ é a expectativa formada hoje da taxa de inflação no período $t+1$ (forward looking) dado o conjunto de informações no período $t$ e $g_{t}$; é o gap produto $\left(y_{t}-y^{*}\right)$ devido à rigidez nominal. A teoria sugere que $\beta$ é aproximadamente 1 e $\delta$ positivo. $O$ último termo não consta na versão de Mankiw e Reis (2002), mas introduzimos este elemento, para permitir que a taxa de inflação também dependa de outras variáveis que não as expectativas e a demanda agregada, como, por exemplo, choques de oferta de preços específicos, como o preço do petróleo.

A relação entre a regra de Taylor e a NKPC decorre da ancoragem das expectativas que o uso da regra de Taylor propicia. Mesmo em um ambiente de elevado grau de rigidez, os eventuais choques suscetíveis na economia que geram um cenário de expectativas voláteis, o comprometimento das autoridades monetárias com o uso de regras que auxiliam na construção de credibilidade, como em Kydland e Prescott (1977), fazendo com que os custos sociais de se combater inflação embutidos na curva Phillips sejam consideravelmente menores. A política monetária, ao fixar uma taxa nominal de juros via regra de Taylor, causará menos recessão, ou em termos da

\footnotetext{
${ }^{7}$ A contribuição desenvolvida em Mankiw e Reis (2002), que segue o percurso já desenvolvido por Calvo (1983) para deduzir uma curva de Phillips com expectativas forward looking, tem o mérito de justificar a validade da curva de Phillips mesmo na presença de expectativas racionais, quando os agentes econômicos, em termos de comportamento microeconômicos, apresentam alguma forma de rigidez nominal de preços. No caso, por um problema de coordenação, somente uma parcela $\lambda$ das firmas ajustam os preços no período $t$. Isso é suficiente para permitir que uma regra de política monetária do tipo Taylor possa afetar a demanda agregada e com isso, via Lei de Okun, a taxa de desemprego. O parâmetro $\beta$, na equação equivale ao parâmetro $\alpha \lambda^{2} /(1-\lambda)$ em Mankiw e Reis.
} 
NKPC, causará um desvio bem menor do produto em relação ao produto potencial. Mas a rigidez nominal de preços, em função de escalonagem ou problema de coordenação dos reajustes de preços por parte das empresas, impende à completa neutralidade da política monetária. $\mathrm{O}$ desvio do produto, com inflação ancorada em expectativas racionais, é menor, mas não nulo.

\subsection{A NKPC E O PROBLEMA DA OFERTA}

A macroeconomia novokeynesiana tem geralmente enfatizado a dinâmica anticíclica da política monetária presente na regra de política monetária. Quando a política monetária reage ao hiato do produto, o primeiro mecanismo privilegiado na maioria dos modelos é a reação da demanda agregada via consumo e investimentos. A demanda agregada pode se deslocar então ao longo da curva da curva de oferta agregada. A curva de oferta tem então uma forma côncava monotônica com inclinação positiva no plano preço-produto, de forma que quanto menor o hiato do produto mais vertical a curva de oferta se torna. No limite, com hiato do produto igual a zero qualquer política econômica teria impacto apenas no nível de preços. Nesses modelos não há possibilidade de deslocamento da curva de oferta como um todo.

Assim, na teoria macroeconômica da determinação do nível de renda, em um regime marcado pelas relações da curva de Phillips combinadas com o uso de regras monetárias do tipo Taylor, as variações na oferta expressam a variação do produto causada pelo deslocamento da demanda apenas, mas não do produto potencial. Dessa feita, tais modelos refletem apenas o efeito demanda do investimento, mas não seu efeito capacidade, como já destacado por Domar (1946, 1947). O “desprezo" pelo efeito capacidade deve-se, sobretudo, aos aspectos de curto "prazismo" dos modelos, sendo que o conceito de curto prazo é exatamente caracterizado pelo fato de que o produto potencial, neste pequeno intervalo de tempo, está dado, ou então, que ele cresce a uma taxa constante.

Isso equivale assumir que o produto potencial é uma variável exógena cuja variação não é afetada pelo próprio ciclo econômico. Essa hipótese de um produto potencial exógeno nos parece uma simplificação por demais drástica, pois na realidade, o que se constata é que a taxa de crescimento do produto potencial varia no tempo, conforme o estágio do ciclo de negócios em que se encontra a economia. O modelo a seguir introduz uma modificação na NKPC que endogeniza o produto potencial.

Desenvolvimentos recentes na literatura novokeynesiana têm procurado incluir o efeito capacidade dos investimentos na dinâmica da inflação. Uma dessas recentes incursões é o trabalho de Razin (2004), que utiliza um modelo novokeynesiano baseado em procedimentos de otimização microfundamentados por parte de consumidores 
representativos e empresas que operam em mercados específicos de bens. A diferenciação (isolamento) de empresas em mercados específicos permite que o modelo trate esses mercados em uma estrutura de concorrência monopolística com fixação de preços escalonadas no tempo (staggered price). Algumas firmas reajustam preços no tempo 1 outras no tempo 2. Isso é possível porque os mercados de trabalhos também são setorizados. Assim, cada setor possui a sua própria curva de oferta e demanda de trabalho, de forma que o salário real (o salário unitário é custo marginal de cada empresa) pode ser rígido em função da defasagem temporal entre os mercados. Além disso, a maior novidade desse modelo está em introduzir uma nova equação para determinação do produto potencial ${ }^{8}$ fazendo com que o mesmo passe a depender não apenas da inflação e do gap do produto, como ocorre normalmente na NKPC (veja equação [v] acima), mas também do estoque de capital. A curva de Phillips de Razin (2004)9 , contém um terceiro elemento que é o produto potencial, assumindo a forma a seguir:

$$
\pi_{t}=\beta E_{t-1} \pi_{t}+\delta\left(y_{t}-y_{t}^{*}\right)-\phi y_{t}^{*}+z_{t}
$$

Note-se que o primeiro termo, que é a presença das expectativas racionais na NKPC, apesar de ser indexado por $t-1$, é de fato expectativas do tipo forward looking, pois se adiantarmos em um período o termo inteiro, obteríamos a expressão $E_{t} \pi_{t+1}$ tal como a equação (v). A equação (vi), portanto, preserva a característica básica da NKPC que é a presença de expectativas racionais do tipo forward looking e rigidez de preços, mas adiciona o efeito capacidade do investimento, que aparece no último termo. Na ausência do efeito capacidade, a curva de Phillips volta à sua forma original, tal como expressa em (v).

\section{UM MODELO DE MÉDIO PRAZO COM NKPC E NÍVEL ÓTIMO DE CAPITAL VARIÁVEL}

Sabendo que a política econômica compreende um mix de políticas monetária, fiscal e cambial, vamos contemplar os efeitos de curto prazo que as variáveis chaves (juros, gastos públicos, tributos e câmbio) exercem sobre a dinâmica de longo prazo em uma economia que tem como regime de política monetária o sistema de metas de inflação.

\footnotetext{
${ }^{8} \mathrm{O}$ produto potencial é aquele nível de produto que se observa quando os preços são completamente flexíveis, de forma que todas as empresas estariam com seus preços corrigidos, sem problemas de coordenação.

${ }^{9}$ A equação (vi) aqui equivale à equação (11) de Razin. Para fins de simplificação aqui, substituímos as expressões algébricas de Razin que representam os parâmetros de cada termo da equação (vi) pelos símbolos $\beta, \delta$ e $\phi$, de forma que possamos manter nossa forma de notação e assim não causar confusão na cabeça do leitor. A novidade aqui está no termo $\phi y^{*}$.
} 
Assim, vamos supor que a função reação que o Banco Central utiliza é uma regra de Taylor, ou seja:

$$
i=\alpha_{1}\left(\pi-\pi^{*}\right)+\alpha_{2}\left(y-y^{*}\right) \alpha_{1}>0, \alpha_{2}>0
$$

Como o nível de produto, dado certo nível tecnológico, é uma proporção $\tau$ do estoque de capital existente $(\tau=y / k)$, podemos assumir que o hiato do produto é determinado pelo diferencial do volume de capital em relação ao volume de capital ótimo:

$$
y-y^{*}=\tau\left(k-k^{*}\right) \tau>0
$$

A oferta agregada é especificada pela curva de Phillips, equivalente à NKPC (v) que foi tratado na seção 2, com expectativas racionais tipo forward looking:

$$
\pi-\omega\left(y-y^{*}\right)+\pi^{e} \omega>0
$$

Podemos então reescrever (3), como base na equação (2), da seguinte forma:

$$
\pi=\omega \tau\left(k-k^{*}\right)+\pi^{e}
$$

A função reação do Banco Central pode ser também reescrita levando (4) e (2) em (1):

$$
i=\tau\left(\alpha_{1} \omega+\alpha_{2}\right)\left(k-k^{*}\right)+\alpha_{1}\left(\pi^{e}-\pi^{*}\right)
$$

A equação (5) descreve o comportamento da política monetária quanto à fixação da taxa de juros. A interpretação correta do primeiro termo é de que a variável $k$ representa o estoque de capital efetivamente utilizado na produção de certo nível de produto, enquanto que $k^{\star}$ é o estoque de capital existente. Isso define o próprio grau de utilização medido não pelo lado do produto, mas pelo lado da mobilização da capacidade instalada. A introdução de uma variável que representa o estoque de capital na curva de Phillips, em substituição à tradicional variável produto (Y), nos permitirá, posteriormente, incorporar o efeito da expansão da capacidade de produção e assim capturar seus efeitos sobre a dinâmica da taxa de inflação. O primeiro termo da equação (5) introduz na função de reação da autoridade monetária a possibilidade de que a política monetária possa levar em conta não apenas as variações do produto pelo lado da demanda, como é realizado tradicionalmente, mas também pelo lado da oferta. $\mathrm{O}$ segundo termo diferencia-se em relação à regra de Taylor na versão original (1), por determinar que a taxa de juro seja maior quanto maior for o desvio das expectativas de inflação $\left(\pi^{e}\right)$ em relação à meta estabelecida pela autoridade monetária $\left(\pi^{\star}\right)$, ao invés de se referir à diferença entre a inflação observada e a meta. 
- Introduzindo o setor externo: em face da existência de um efeito pass-trough podemos assumir que o impacto de variações cambiais sobre a taxa de inflação esperada $\left(\pi^{e}\right)$ é dada por:

$$
\pi^{e}=\xi_{1} \varepsilon+\xi_{2} \pi^{*} \quad \xi_{1}>0, \xi_{2}>0
$$

em que $\varepsilon$ é a taxa real de câmbio dada por $\varepsilon=E P^{\star} / P$. Resolvendo (6) para e obtemos, e fazendo $\phi_{1}=1 / \xi_{1}$ e $\phi_{2}=\xi_{2} / \xi_{1}$ obtemos:

$$
\varepsilon=\phi_{1} \pi^{e}-\phi_{2} \pi^{*} \quad \phi_{1}>0, \phi_{2}>0
$$

- Demanda agregada: no que concerne às variáveis que representam as possibilidades de afetar a demanda agregada pelo lado das políticas fiscais, o modelo assume que o setor público opera sob uma restrição orçamentária que o impede de elevar continuamente a dívida pública tal que:

$$
\dot{B}=i B+G-T
$$

em que $B$ representa os títulos públicos, $G$ os gastos públicos e $T$ são os tributos. Assumiremos em (8) duas hipóteses: a primeira é que a política fiscal busca preservar a sustentabilidade da dívida pública, de modo que $\dot{B}=0$; a segunda hipótese preserva o caráter anticíclico da política fiscal, em que os gastos do governo são determinados a partir da flutuação do produto, ou seja:

$$
G=g\left(y-y^{*}\right)
$$

Com isso, a restrição do setor público pode ser dada pela seguinte equação:

$$
T=g\left(y-y^{*}\right)+i B
$$

Levando a função reação do Banco Central, especificada pela equação (5), e a equação (2) em (10), após coletar termos, podemos determinar os tributos da seguinte forma:

$$
T=\left[g+\left(a_{1} \omega+a_{2}\right) \tau B\right]\left(k-k^{*}\right)+a_{1} B\left(\pi^{e}-\pi^{*}\right)
$$

Esta equação mostra que a política fiscal, tanto pelo lado dos gastos quanto pelo lado da arrecadação, se ajusta para equilibrar o orçamento quando as taxas de juros variarem por ocasião da reação da política monetária via regra de Taylor estendida, tal como definida em (5). Isso equivale afirmar que a política fiscal se ajusta e se coaduna à política monetária, atuando ambas de forma coordenada. Nesse aspecto, este modelo permite que tenhamos políticas fiscais e monetárias harmônicas, mesmo em uma estrutura analítica keynesiana de caráter anticíclico. 
Até aqui conseguimos contemplar as equações que determinam no curto prazo as variáveis-chave do nosso modelo que compreende o mix de políticas monetária, cambial e fiscal.

Tendo em vista que no curto prazo $i, \pi, \varepsilon$ e $T$ podem sofrer choques das variáveis $k$ e $\pi^{e}$, o efeito desses choques pode determinar a estabilidade do modelo de sua dinâmica em longo prazo. Para verificar isso, a equação que determina a demanda agregada $y$, pode ser decomposta da seguinte forma:

$$
y=c+\dot{k}+G+x
$$

em que $c$ é o consumo agregado, $I=\dot{k}^{10}$ é o investimento, $G$ o gasto público e $x$ a exportação líquida. Assumindo que os componentes da demanda possuem as seguintes especificações:

$$
\begin{gathered}
c=c_{1}(y-T) c_{1}>0 \\
G=g\left(y-y^{*}\right)=g \mathrm{t}\left(k-k^{*}\right) \\
c=c_{1}(y-T) c_{1}>0 \\
G=g\left(y-y^{*}\right)=g \mathrm{t}\left(k-k^{*}\right) \\
\mathrm{x}=\mathrm{de} \quad \mathrm{d}>0
\end{gathered}
$$

e levando o conjunto de equações (13) em (12) e resolvendo para $\dot{k}$, obtemos a função investimento:

$$
\dot{k}=y-c_{1}(y-T)-g \tau\left(k-k^{*}\right)-\delta \varepsilon
$$

A equação (14) representa a dinâmica de longo prazo da variação da oferta agregada, a partir dos determinantes de curto prazo da demanda agregada. De modo mais preciso, representa a variação do estoque de capital necessário para atender a demanda existente, o qual pode ser maior ou menor do estoque total de capital disponível $\left(k^{*}\right)$. O modelo preserva assim seus traços keynesianos, na medida em que a oferta pode se deslocar em longo prazo, em função de choques de demanda em curto prazo.

- Produto Potencial pró-cíclico: a maioria dos modelos macroeconômicos de oferta e demanda trata o produto potencial como uma constante. Independentemente de qual seja o estágio de um ciclo econômico que esteja sendo percorrido pela economia, o produto potencial varia a uma taxa constante. No entanto, estudos empíricos (Barbosa Filho, 2005) têm apontado que o produto potencial varia ao longo do ciclo econômico, e mais que isso, ele o faz de forma pró-cíclica. Isto é, quando a economia está crescendo o produto potencial se expande a uma taxa maior e quando

\footnotetext{
${ }^{10} \mathrm{~A}$ variável $\dot{k}$ é a variação do estoque de capital em relação à variável tempo $t, \partial k / \partial t$.
} 
a economia está em desaceleração a uma taxa menor. A consequência desse comportamento é que a relação entre grau de utilização da capacidade produtiva ou o hiato do produto sobre os preços é menor do que o previsto na maioria dos modelos. Nos momentos de crescimento as restrições da oferta sobre a inflação são atenuadas pela resposta flexível do produto potencial e a inflação tende a ser menor. Da mesma forma, nos momentos de desaceleração ou recessão, a queda do produto potencial reduz o efeito da retração da demanda efetiva atenuando a tendência à deflação, nessa fase do ciclo econômico. Para introduzir esse efeito no modelo podemos representar o comportamento do produto potencial $\left(K^{*}\right)$ como dependendo de forma contínua da demanda agregada $(K)$ e de dois coeficientes expectacionais $(\alpha, \beta)$ que variam conforme o estágio do ciclo econômico, com base nas equações abaixo:

$$
\begin{aligned}
& K_{t}^{*}=K_{t}^{\alpha+\beta}+\mu_{t} \\
& k^{*}=(\alpha+\beta) k
\end{aligned}
$$

A equação (15b) é obtida tomando-se o logaritmo natural de (15a), com o que obtém sua forma linear. $\mathrm{O}$ coeficiente $\alpha$ representa o efeito tendência do capital, ou o componente inercial. Se a economia vem de uma trajetória de crescimento acelerado a assume um valor elevado. $O$ coeficiente $\beta$ representa o componente cíclico do capital e é influenciado pela expectativa futura dos agentes quanto à demanda futura. Se as expectativas foram otimistas $\beta$ será alto. O gráfico 1 traz quatro situações possíveis para a trajetória de $k^{*}$ no tempo em função dos valores dos parâmetros $\alpha$ e $\beta$. A esquerda do ponto $T$ no tempo, a tendência da trajetória do nível de capital ótimo é determinada pelo parâmetro $\alpha$, dado que esse parâmetro reflete o efeito tendência do capital. A direita do ponto $T$, a trajetória do nível ótimo de capital é determinada pelo valor do parâmetro $\beta$.

A ideia é mostrar que a trajetória do nível de capital ótimo depende do conjunto dos valores $\alpha$ e $\beta$. Como $\alpha$ é o componente inercial, com um valor maior que 1 a tendência do nível de capital ótimo será crescente até o ponto $T$ no tempo; a partir desse ponto a tendência do nível de capital ótimo dependerá das expectativas acerca da demanda, expressa pelo parâmetro $\beta$, caso seu valor supere a unidade, a tendência do nível de capital ótimo permanece crescente.

Substituindo (15b) em (12) podemos representar a função investimento ampliada:

$$
\dot{k}=y-c_{1}(y-T)-g \tau[1-(\alpha+\beta) k]-\delta \varepsilon
$$

A equação que determina a demanda agregada pode ser obtida substituindo o conjunto de equações (13) e a equação (16) em (12), com o que obtemos:

$$
\dot{k}=\left(1-c_{1}\right)+c_{1}\left[\left(a_{1} w+a_{2}\right)(1-\alpha-\beta) B\right] \tau k+\left[c_{1} a_{1} B-\delta \varphi_{1}\right] \pi^{e}+\left(\delta \varphi_{2}-c_{1} a_{1}\right) \pi^{*}
$$




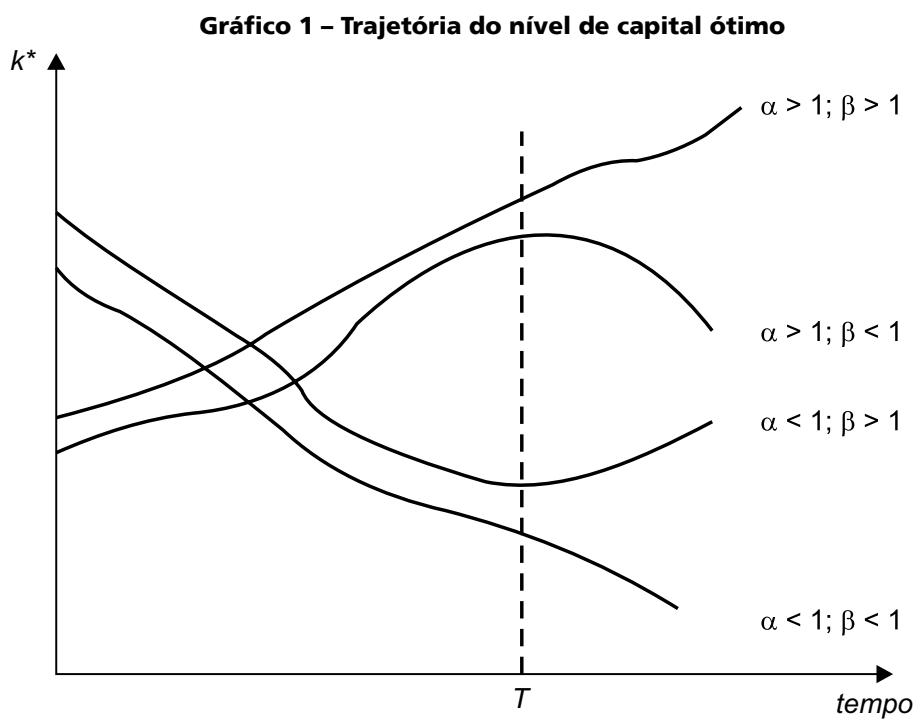

Fonte: Elaboração própria.

- A curva de Phillips expandida e deslocamentos da oferta: a equação (3b) mostrou o equilíbrio de curto prazo da inflação, de modo que podemos reescrever essa equação da seguinte forma:

$$
\pi=\omega \tau k-\omega \tau k^{*}+\pi^{e}
$$

A equação (4) é a curva de Phillips. A partir dela, podemos obter o seguinte resultado de estática comparativa:

$$
\frac{\partial \pi}{\partial k^{*}}=-\omega \tau<0
$$

De acordo com o resultado em (19) podemos observar que expansões no nível de capital ótimo $k^{*}$, proporcionam reduções no nível de inflação. Esse resultado pode ser visto de acordo com o gráfico 2 a seguir.

Com a inserção do nível de capital ótimo podemos verificar que há possibilidade de deslocamento da curva de oferta como um todo, de $\mathrm{OA}_{0}$ para $\mathrm{OA}_{2}$ no gráfico 2, de modo que expansões no nível de capital ótimo $k^{*}$, proporcionam reduções no nível de inflação. 
Gráfico 2 - Curva de Phillips e deslocamentos da oferta

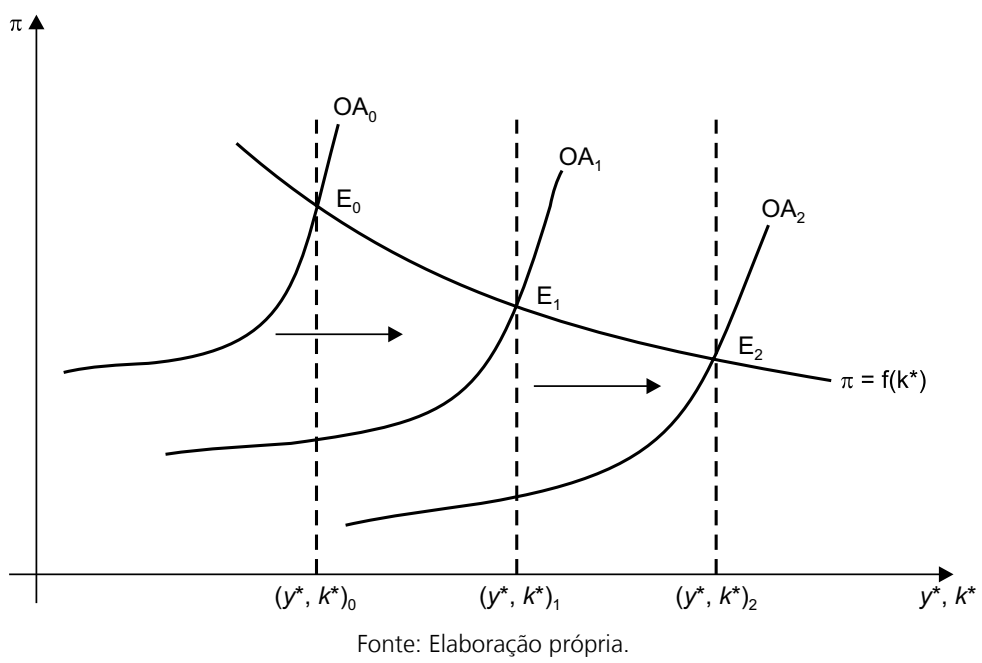

- Dinâmica de Longo Prazo e Equilíbrio: em longo prazo $\pi^{e}$ se comporta de acordo com a seguinte equação diferencial homogênea:

$$
\frac{d \pi^{e}}{d t}=\sigma\left(\pi-\pi^{e}\right) \quad \sigma>0
$$

em que a variação da inflação esperada no tempo é dada pelo diferencial da inflação efetiva em relação a própria inflação esperada.

Sabendo que a equação (17) reflete a dinâmica de longo prazo da demanda agregada o equilíbrio em longo prazo pode ser analisado com base na seguinte matriz jacobiana:

$$
\left[\begin{array}{cc}
\left\{\left(a_{1} \omega+a_{2}\right)(1-\alpha-\beta) B\right\} \tau & \left(c_{1} a_{1} B-\delta \varphi_{1}\right) \\
\sigma \omega \tau(1-\alpha-\beta) & 0
\end{array}\right]
$$

$\mathrm{O}$ traço e o determinante da matriz jacobiana são dados por:

$$
\begin{gathered}
\text { Traço }=\left\{\left(a_{1} \omega+a_{2}\right)(1-\alpha-\beta) B\right\} \tau \\
\text { Determinante }=-\sigma \omega \tau(1-\alpha-\beta)\left(c_{1} a_{1} B-\delta \varphi_{1}\right)
\end{gathered}
$$

As condições para o sistema (17) e (20) assumir uma trajetória globalmente estável exige que o traço da matriz jacobiana seja menor que zero e o seu determinante maior que zero. 
Para que o traço seja menor que zero e o determinante maior que zero, temos que impor a seguinte condição: $\alpha+\beta>1$.

Essa condição de estabilidade justifica a necessidade de tratarmos o produto potencial como uma variável endógena, uma vez que a expansão da capacidade produtiva da economia depende da própria taxa de investimento observada ao longo do ciclo econômico. Não faz muito sentido imaginar que a velocidade de expansão da capacidade produtiva de um período recessivo seja a mesma de uma fase de expansão robusta. Alguns métodos empíricos ${ }^{11}$ estimam o produto potencial através da aplicação de filtros suavizadores de séries históricas, isolando o componente de tendência do componente cíclico, tal como médias móveis ou filtros como de Hodrick e Prescott (1997), etc. A estimação do produto potencial não é uma tarefa trivial e existem diversos métodos, muitos baseados no conceito de Produtividade Total dos Fatores (PTF), que leva em conta não apenas variações no volume do estoque de capital, mas também o aumento da produtividade. De qualquer forma, o importante para nosso objetivo atual é a evidência empírica de que o produto potencial tem um comportamento pró-cíclico em curto prazo e é endógeno em longo prazo (Barbosa Filho, 2005, p. 55 e figura 6, p. 68).

Uma curva de Phillips que leve em conta o comportamento pró-cíclico e endógeno ao mesmo tempo, deveria ser obtida a partir da transformação do termo $y^{*}$ ou $k^{*}$ por um componente que expresse seu comportamento dinâmico ao longo dos ciclos. Uma maneira de fazer isso é assumir o efeito capacidade do investimento de Domar (1946), de forma que a expansão do estoque de capital será endógena. O resultado da análise de estabilidade do modelo impõe a condição $\alpha+\beta>1$. Tal resultado pode ser associado aos três fatos estilizados por Mishkin (2007), quais sejam: (i) a persistência dos choques de inflação estão declinando; (ii) a curva de Phillips esta se tornando mais horizontal e; (iii) a inflação tem se mostrado menos reativa à choques nos preços. Assim, a condição $\alpha+\beta>1$ associa-se a expansão da capacidade do estoque de capital induzir deslocamentos da curva de oferta ao longo do tempo, o que permite a ocorrência de menos choques de inflação, dado a presença de uma curva de Phillips cada vez mais horizontal.

Vale destacar que o desenvolvimento de modelos que levem em consideração uma regra para a fixação da taxa de juros, uma relação inversa entre inflação e hiato do produto, o equilíbrio no mercado de bens, o movimento das expectativas inflacionárias e o movimento da taxa de câmbio, mesmo considerando algumas hipóteses restritivas, é algo relevante quando se trata de uma pesquisa teórica em macroeconomia, haja vista quando se misturam relações de longo prazo, como o modelo de Domar (1946), com

\footnotetext{
${ }^{11}$ Para uma visão sobre diversos métodos adotados para estimar o produto potencial no Brasil ver Silva Filho (2001) e Barbosa Filho (2005).
} 
relações de curto prazo (mesmo sabendo que o modelo de Domar segue uma tradição de modelagem keynesiana).

Quando se trabalha com modelos macrodinâmicos temos que levar em consideração que as variáveis de interesse (endógenas) tendem ao equilíbrio em longo prazo ou não, posto que as condições para a estabilidade de um dado sistema pode sugerir alguma prescrição relevante para a política econômica ou até mesmo algum requisito para a parametrização das constantes definidas em algumas equações comportamentais preestabelecidas.

\section{CONSIDERAÇÕES FINAIS}

O objetivo do artigo foi propor uma explicação adicional para a horizontalização da curva de Phillips de acordo com os fatos estilizados por Mishkin (2007).

$\mathrm{O}$ artigo introduziu além da ancoragem da inflação por um processo de expectativas racionais do tipo forward looking, uma explicação adicional para a dinâmica da inflação-produto é o efeito da capacidade de produção, de modo que isso contribui para o deslocamento da curva de oferta agregada mesmo em médio prazo, ao invés de considerar apenas o deslocamento da oferta demanda agregada ao longo da curva, como tradicionalmente ocorre. Assim, a principal característica do modelo foi introduzir uma modificação na curva de Phillips, que passa a responder, não apenas ao hiato do produto, mas também à ampliação do produto potencial, o que é representado por sua proxy, o estoque de capital.

Em comparação com o arcabouço da teoria macroeconômica novokeynesiana, que mesmo usando o conceito de gap ou hiato do produto, esta se restringe aos efeitos da demanda agregada prestando pouca atenção aos efeitos da oferta sobre a inflação.

A inserção do nível de capital ótimo mostrou que é plausível o deslocamento da curva de oferta como um todo, de modo que expansões no nível de capital ótimo $k^{*}$, geram reduções no nível de inflação.

Assim, o resultado do modelo apresentado corrobora com a hipótese de que um produto potencial exógeno, que se reflete também em um nível de capital exógeno, nos parece uma simplificação por demais drástica, como é feito no arcabouço NKPC, uma vez que ao considerarmos uma dinâmica para a trajetória do capital, podemos verificar os efeitos de uma mudança na taxa de crescimento do produto potencial, via mudanças no nível de capital ótimo, sobre a trajetória da inflação. 


\section{REFERÊNCIAS}

AKERLOF, G.A.; YELLEN, J.L. A near-rational model of the business cycle with wage and price inertia, Quarterly Journal of Economics, v. 100, Supplement, p. 823-838, 1985.

BARBOSA FILHO, N.H. Estimating potential output: a survey of alternative methods and their applications to Brazil, Texto para discussão - IPEA, n. 1.092, 2005.

BLANCHARD, O.; KIYOTAKI, N. Monopolistic competition and the effects of aggregate demand, American Economic Review, v. 77, September, p. 647-666, 1987.

BLANCHARD, O. Macroeconomics. Londres: Prentice-Hall, 1997.

BLINDER A.S.; SOLOW, R.M. Does fiscal policy matter? Journal of Public Economics, v. 2, p. 319-337, 1973.

BUITER, W.H. 'Crowding out' and the effectiveness of fiscal policy, Journal of Public Economics, v. 7, p. 309-328, 1977.

CALVO, G.A. Staggered prices in a utility maximization framework, Journal of Monetary Economics, v. 12, p. 383-398, 1983.

CECCHETTI, S.G.; HOOPER, P.; KASMAN, B.C.; SCHOENHOLTZ, K.L.; WATSON, M.W. Understanding the evolving inflation process. In: U.S. MONETARY POLICY FORUM, Washington, D.C., March 9, 2007.

CLARIDA, R.; GALI, J.; GERTLER, M. The science of monetary policy: a new Keynesian perspective. Journal of Economic Literature, v. 37, December, p. 1.661-707, 1999.

COGLEY, T.; SARGENT, T. Drifts and volatilities: Monetary policies and outcomes in the Post WWII U.S., Review of Economic Dynamics, v. 8, April, p. 262-302, 2005.

DOMAR, E.D. Capital expansion, rate of growth and employment, Econometrica, v. 37, n. 1, p. 137-147, 1946.

DOMAR, E.D. Expansion and employment, American Economic Review, p. 34-55, 1947.

FRIEDMAN, M. The role of monetary policy, American Economic Review, v. 58, p. 1-17, 1968. . Comments on the critics, Journal of Political Economy, v. 80, p. 906-950, 1972.

. Nobel lecture: Inflation and unemployment. Journal of Political Economy, v. 85, n. 3 , p. $451-472,1977$.

GALI, J.; GERTLER, M. Inflation dynamics: a structural econometric analysis, Journal of Monetary Economics, v. 44, p. 195-222, 1999.

HODRICK, R.J.; PRESCOTT, E.C. Postwar US business cycles: An empirical investigation. Journal of Money, Credit and Banking, v. 29, p. 1-16, 1997.

KALDOR, N. The scourge of monetarism. Oxford: Oxford University Press, 1982.

KYDLAND, F.E.; PRESCOTT, E.C. Rules rather than discretion: The inconsistency of optimal plans, Journal of Political Economy, v. 27, p. 129-47, 1977.

LUCAS, R.E. Econometric testing of the natural rate hypothesis. In: ECKSTEIN, O. (ed.) The econometrics of price determination Conference. Washington, D.C, Board of Governors of the Federal Reserve System, 1972 (reprinted in 1981), p. 90-103. 
Some international evidence on output-inflation tradeoffs. American Economic Review, v. 63, p. 326-334, 1973.

Econometric policy evaluation: a critique. Rochester Conference Series, Carnegie, v. 1, p. 19-46.

. Studies in business-cycle theory. Cambridge, MA: MIT Press, 1981.

MANKIW, N.G. Small menu costs and large business cycles: a macroeconomic model of monopoly, Quarterly Journal of Economics, v. 100, n. 2, May, p. 529-539, 1985.

MANKIW, N.G.; REIS, R. Sticky information versus sticky prices: a proposal to replace the new Keynesian Phillips curve, Quarterly Journal of Economics, v. 117, n. 4, November, p. 1.295-1.328, 2002.

MENON, J. Exchange rate pass-through. Journal of Economic Survey, v. 9, n. 2, p. 197-231, 1995.

MISHKIN, F.S. Inflation dynamics, NBER Working Paper, n. 13147, June, 2007.

MOORE, B. Horizontalist and verticalist. Cambridge: Cambridge University Press, 1988.

MUTH, J.F. Rational expectations and the theory of price movements. Econometrica, v. 29, n. 3, p. 315-335, Junho, 1961.

PHELPS, E. Money-wage dynamics and labor-market equilibrium, Journal of Political Economy, v. 68, part II, August, p. 678-711, 1968.

PHILLIPS, W.A. The relation between unemployment and the rate of change of money wages in the United Kingdom, 1861-1957, Economica, v. 25, Novembro, 1958.

RAZIN, A. Aggregate supply and potential output, Working Papers - Hong Kong Institute for Monetary Research, n. 142004, 2004.

SAMUELSON, P.A.; SOLOW, R.M. Analytical aspects of anti-inflation policy. American Economic Review, v. 50, n. 2, May, p. 177-194, 1960.

SARGENT, T.; WALLACE, N. Rational expectations, the optimal monetary instrument, and the optimal money supply rule. Journal of Political Economy, v. 83, n. 2, p. 241-254, 1975.

SBORDONE, A. Prices and unit labor costs: anew test of price stickness, Journal of Monetary Economics, v. 49, p. 265-292, 2002.

SILVA FILHO, T.N. Estimando o produto potencia brasileiro: uma abordagem de função de produção, Trabalhos para discussão - Banco Central do Brasil, n. 17, 2001.

SOLOW, R.M. On theories of unemployment, American Economic Review, v. 70, n. 1, March, p. 1-11, 1980.

STIGLITZ, J.; BRUCE, G. Rumo a um novo paradigma em economia monetária. São Paulo: Francis, 2003.

STOCK, J.; WATSON, M. Why has U.S. inflation become harder to forecast? Journal of Money, Credit, and Banking, v. 39, February, p. 3-34, 2007.

TAYLOR, J.B. Staggered wage setting in a macro model. American Economic Review, v. 69, p. 108-113, 1979. 
Aggregate dynamics and staggered contracts. Journal of Political Economy, v. 88, p. 1-24, 1980.

Discretion versus policy rules in practice. Carnegie-Rochester Conf. Serv. Public Policy, v. 39, p. 195-214, 1993.

TOBIN, J; BUITER, W.H. Long run effects of fiscal and monetary policy on aggregate demand. In: STEIN, J.L. (ed.) Monetarism. Amsterdam: North Holland, p. 273-309, 1976. 